\title{
170-GHz Transceiver with On-Chip Antennas in SiGe Technology
}

\author{
E. Laskin ${ }^{1}$, K.W. Tang ${ }^{1}$, K.H.K. Yau ${ }^{1}$, P. Chevalier ${ }^{2}$, A. Chantre ${ }^{2}$, B. Sautreuil ${ }^{2}$, S.P. Voinigescu ${ }^{1}$ \\ 1) Edward S. Rogers Sr. Dept. of ECE, University of Toronto, Toronto, ON M5S 3G4, Canada \\ 2) STMicroelectronics, 850 rue Jean Monnet, F-38926, Crolles, France
}

\begin{abstract}
A single-chip transceiver with on-die transmit and receive antennas, $R x$ and $T x$ amplifiers, $165-\mathrm{GHz}$ oscillator and static frequency divider is reported in a SiGe HBT process with $\mathbf{f}_{\mathrm{T}} / \mathbf{f}_{\mathrm{MAX}}$ of $270 \mathrm{GHz} / 340 \mathrm{GHz}$. This marks the highest frequency transceiver in silicon and the highest level of functional integration above $100 \mathrm{GHz}$ in any semiconductor technology. The downconversion gain peaks at $-5 \mathrm{~dB}$ and the transmit power is $-5 \mathrm{dBm}$ when measured at the transceiver pads. Both degrade by approximately $25 \mathrm{~dB}$ when measured above the antennas of the transceiver with on-die antennas. The experimental performance of dipole (with and without floating metal strips) and patch antennas is also investigated. The measured 15-dB gain of a standalone amplifier is centered at $170 \mathrm{GHz}$ and remains higher than $10 \mathrm{~dB}$ from $160 \mathrm{GHz}$ to $180 \mathrm{GHz}$ while the saturated output power is $0 \mathrm{dBm}$ at $165 \mathrm{GHz}$.
\end{abstract}

Index Terms - SiGe HBT technology, mm-wave transceivers, integrated antenna, amplifiers, frequency divider

\section{INTRODUCTION}

The increase in the cutoff and oscillation frequencies of silicon transistors has continued unabated, allowing for singlechip transceivers to break the $100-\mathrm{GHz}$ barrier [1]. This opens up opportunities for new, very-short range imagers for dental and medical applications where sub-mm resolution is required over distances of several centimeters. Although $100+\mathrm{GHz}$ systems on chip are now feasible at room temperature, one of the significant hurdles that remain is posed by packaging. The flip-chip pad capacitance and design rules continue to remain too large and too coarse to be useful above $100 \mathrm{GHz}$ in silicon technologies with a conventional thin dielectric and thin-metal BEOL. Although antennas with $+2 \mathrm{dBi}$ gain have been demonstrated in silicon ICs at $77 \mathrm{GHz}$ [2], the multi-chip packaging and the lens required to achieve such performance fall short of the packaging-free promise of on-die antennas. In this paper we explore three different $170-\mathrm{GHz}$ antenna designs for integration in a digital 130-nm CMOS backend and we demonstrate successful operation of a single-chip transceiver which includes two antennas and yet satisfies the strict metal density rules of $130-\mathrm{nm}$ SiGe BiCMOS production technology.

\section{TRANSCEIVER ARCHITECTURE}

The transceiver block diagram is shown in Fig. 1. It re-uses the 165-GHz push-push differential oscillator and Gilbert-cell mixer of [1], along with a new 160-to-180 GHz amplifier, the static frequency divider from [3], and receive and transmit antennas. Three identical amplifiers are included on the transmit, receive, and LO paths, respectively, to significantly improve the transmitter output power and the receiver downconversion gain when compared to the circuit presented in [1].

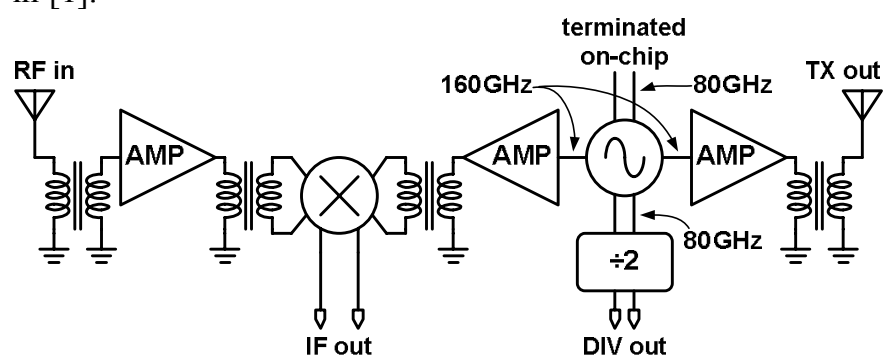

Fig. 1. 160-to-180 GHz transceiver block diagram.

Transformers are employed at the interfaces between circuit blocks to provide good isolation by separating the bias supply planes, and for single-ended to differential conversion. One of the two $80-\mathrm{GHz}$ differential outputs of the oscillator drives an on-die divider [3] to demonstrate the feasibility of integrating an on-chip PLL together with the LO distribution network.

\section{AMPLIFIER DESIGN}

A frequency-scaled version of the five-stage $140-\mathrm{GHz}$ amplifier in [1] was designed to operate in the $160-\mathrm{GHz}$ to $180-\mathrm{GHz}$ range. Its schematic is illustrated in Fig. 2 and consists of three cascode stages followed by two commonemitter stages. The last stage is composed of two devices, with CBEBC layout configuration, connected in parallel to achieve higher output power. The input and output matching networks of the three amplifiers are modified so as to allow for maximum power transfer from the oscillator, to the mixer, or to and from the $50-\Omega$ antenna impedance, depending on where the amplifier is located in the block diagram of Fig. 1.

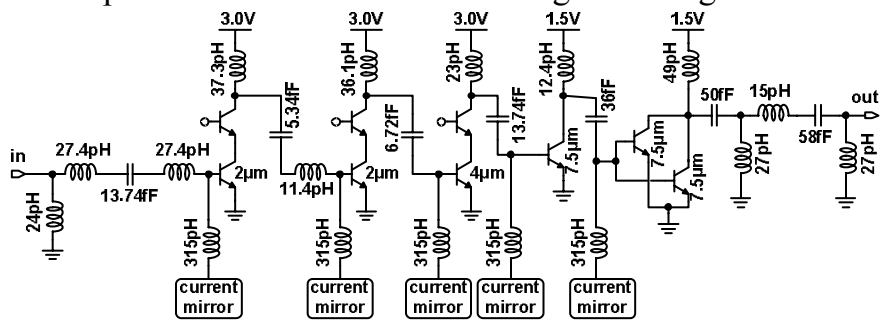

Fig. 2. 160-to-180 GHz amplifier schematic.

Scaling this amplifier in frequency from $140 \mathrm{GHz}$ to $170 \mathrm{GHz}$ was accomplished by adjusting component values and reducing inductive, resistive, and capacitive layout 
parasitics. To tune all the resonant load circuits in all amplifier stages to $170 \mathrm{GHz}$, capacitors with values smaller than $30 \mathrm{fF}$ are required. Since such small MiM capacitor values were not available in the design kit, custom Metal-Oxide-Metal capacitors were designed using ASITIC. Fig. 3 illustrates the 3 -D capacitor structure and the $2 \pi$ model which was used in circuit simulations. The $2 \pi$ equivalent circuit was derived from the simple- $\pi$ circuit employing a procedure similar to that described in [4]. To minimize their inductance and the die area of the amplifier, all capacitors have a short aspect ratio, instead of the square layout typical of MiM capacitors.

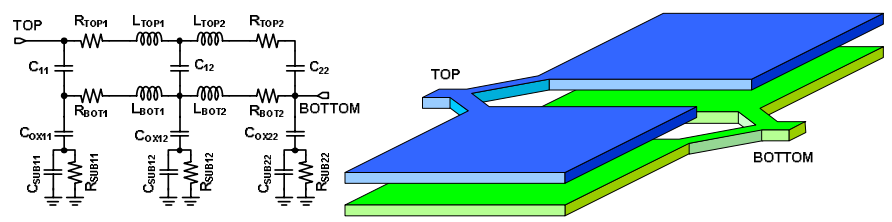

Fig. 3. Custom capacitor layout geometry (right, not to scale) and the corresponding $2 \pi$ equivalent circuit (left).
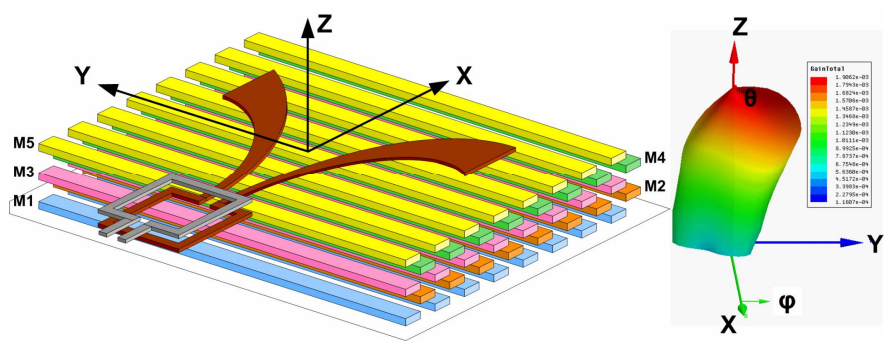

Fig. 4. 3-D view of the dipole antenna with metal fill (left, not to scale). HFFS-simulated radiation pattern of the antenna without metal fill (right).

\section{ANTENNA DESIGN}

In an effort to explore the merits of integrating $170-\mathrm{GHz}$ antennas on chip, three different antennas were designed using HFSS with the goal of minimizing area without sacrificing gain. The first is a patch antenna, realized in the $6^{\text {th }}$ copper layer of the technology, and is used as a reference for size $(475 \mu \mathrm{m} \times 450 \mu \mathrm{m})$ and gain $(-8 \mathrm{dBi}$ in simulations). The second design is a tapered dipole antenna with increased gain and smaller size. Neither of these two designs satisfies the metal density rules of the $130-\mathrm{nm}$ SiGe BiCMOS process and therefore cannot be integrated in a real product. A second dipole-antenna design was then derived from the first by inserting a floating metal-strip pattern under the antenna, similar to a slow-wave t-line [5]. A detailed drawing of the third antenna with metal fill is shown in Fig. 4. The staggered floating metal strips in metals 1 through 5 reduce the dielectric height above the silicon substrate and increase the effective permittivity, thus dramatically reducing the antenna size required for operation at $170 \mathrm{GHz}$. The small size of this antenna and the floating metal under it, ensure its full compliance with the metal-density rules of any modern nanoscale CMOS or SiGe BiCMOS process.

\section{FABRICATION AND TEST PROCEDURE}

The transceiver with and without antennas, and breakouts of the three different antennas and of the amplifier were fabricated in a SiGe HBT technology [6] with $f_{T}$ of $270 \mathrm{GHz}$ and $f_{M A X}$ of $340 \mathrm{GHz}$. The measured $f_{T}$ and $f_{M A X}$ are plotted in Fig. 5 at $25^{\circ} \mathrm{C}$ and $125^{\circ} \mathrm{C}$. The process features 6 copper layers and an aluminum cap layer used for pad openings, MiM capacitors, and poly resistors. The top $\mathrm{Cu}$ layer is $0.9-\mu \mathrm{m}$ thick and $4.5 \mu \mathrm{m}$ above the silicon substrate. $\mathrm{SiO}_{2}$ is employed as the inter-metal dielectric. Die photos of the transceiver, amplifier breakout, and antenna breakouts are shown in Figs. 6-8.

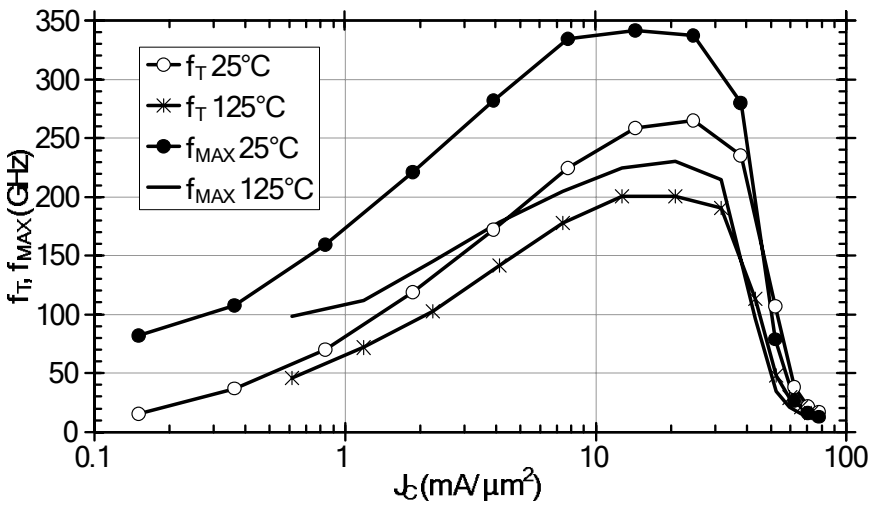

Fig. 5. Measured $f_{T}$ and $f_{M A X}$ vs. current density and temperature for an HBT with an effective emitter size of $0.11 \mu \mathrm{m} \times 5.55 \mu \mathrm{m}$.

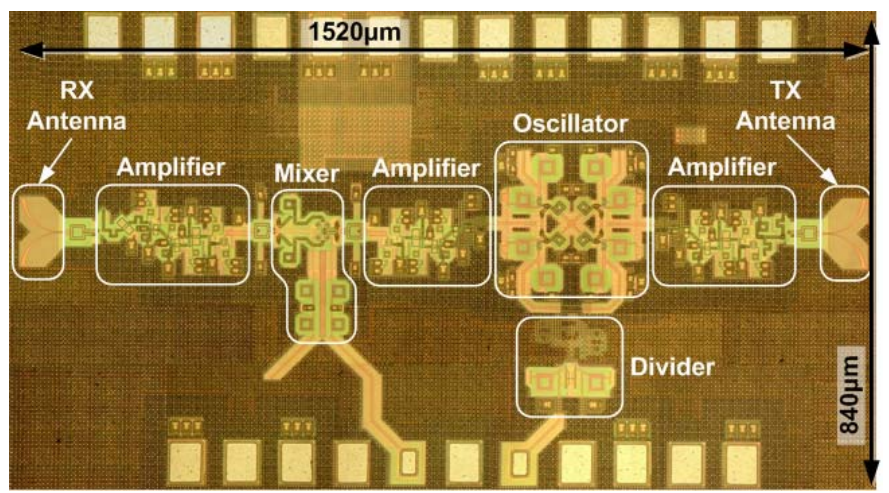

Fig. 6. Die photo of the transceiver with integrated antennas.

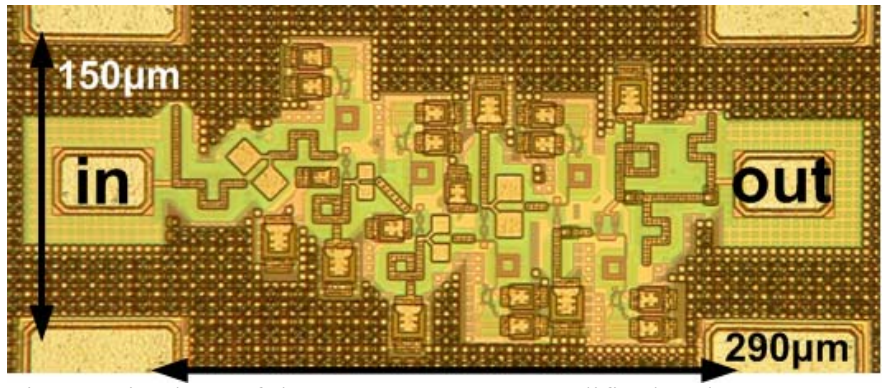

Fig. 7. Die photo of the 160 -to-180 GHz amplifier breakout.

All circuits and the antennas were measured on wafer, in a D-band waveguide setup. The RF input signal was generated using an Oleson multiplier. Output signals in the D-band were measured with an external down-convert mixer and an Agilent 
PSA, or with an Elva D-band power sensor. The antenna radiation pattern was estimated by applying a signal to the input pad of each antenna test-structure and employing a suspended 110-to-170 GHz Cascade Microtech GSG probe to measure the radiated power. All setup losses were deembedded from the measurements. Where applicable (amplifier and transceiver without antennas), the pad capacitance was not de-embedded.

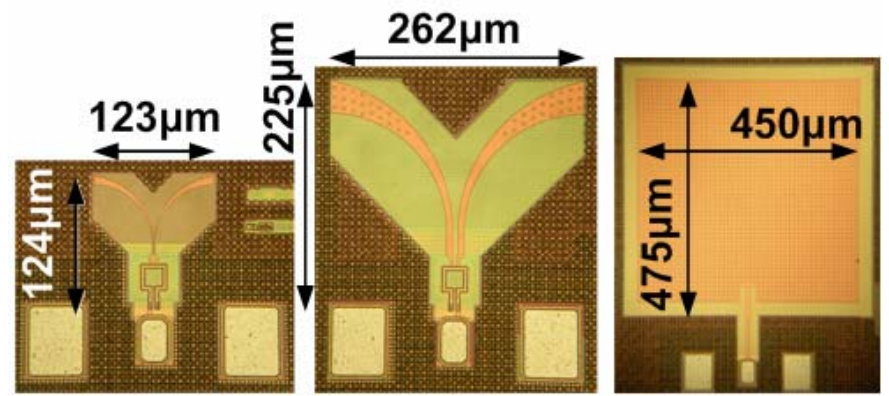

Fig. 8. On-chip antenna test-structures. Left: dipole with fill; center: dipole without metal fill; right: patch antenna (not to scale).

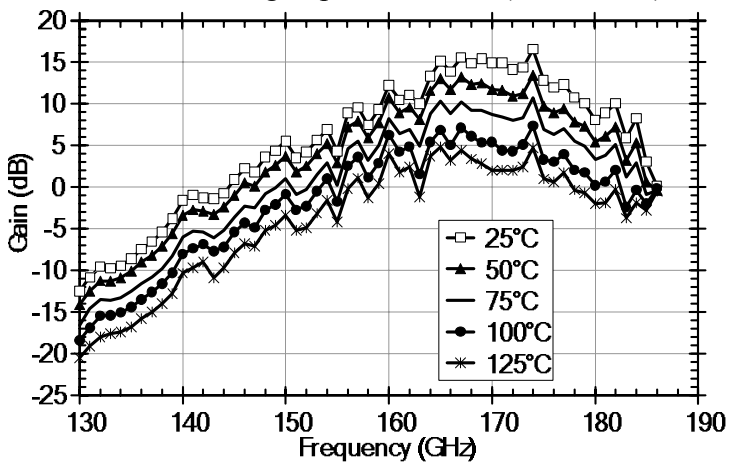

Fig. 9. Measured amplifier gain vs. frequency over temperature.

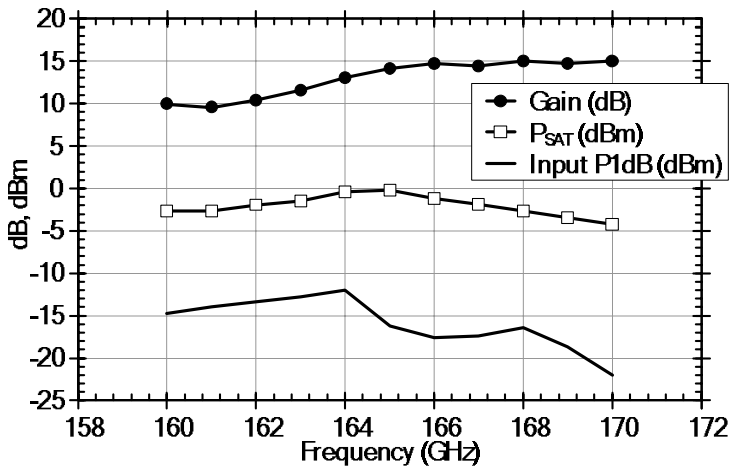

Fig. 10. Measured gain, saturated output power, and input $\mathrm{P}_{1 \mathrm{~dB}}$ of the amplifier at $25^{\circ} \mathrm{C}$.

\section{MEASUREMENT RESUlTS}

\section{A. 160-GHz to 180-GHz Amplifier}

Fig. 9 shows the amplifier gain measured over temperature up to $125^{\circ} \mathrm{C}$. It is higher than $15 \mathrm{~dB}$ from $166 \mathrm{GHz}$ to $174 \mathrm{GHz}$ and the $3-\mathrm{dB}$ bandwidth exceeds $10 \mathrm{GHz}$. Fig. 10 summarizes the amplifier linearity measurements in the 160 -to- $170 \mathrm{GHz}$ range at $25^{\circ} \mathrm{C}$. The maximum output power of $0 \mathrm{dBm}$ occurs at 164 to $165 \mathrm{GHz}$ and the best input $\mathrm{P}_{1 \mathrm{~dB}}$ is
$-12 \mathrm{dBm}$ at $164 \mathrm{GHz}$. The amplifier DC power consumption is $135 \mathrm{~mW}$.

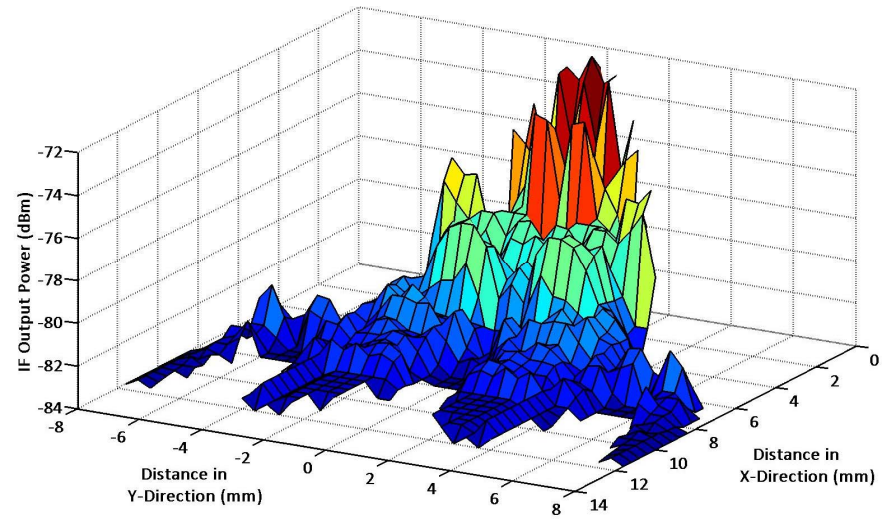

Fig. 11. Antenna pattern measured directly above the receiver.

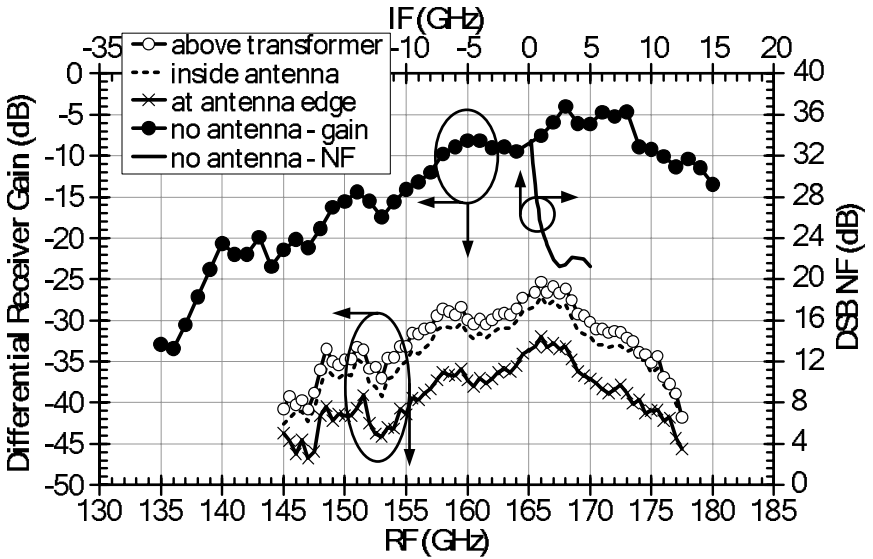

Fig. 12. Measured conversion gain of the receiver with and without antenna, and measured DSB NF of the receiver without antenna.

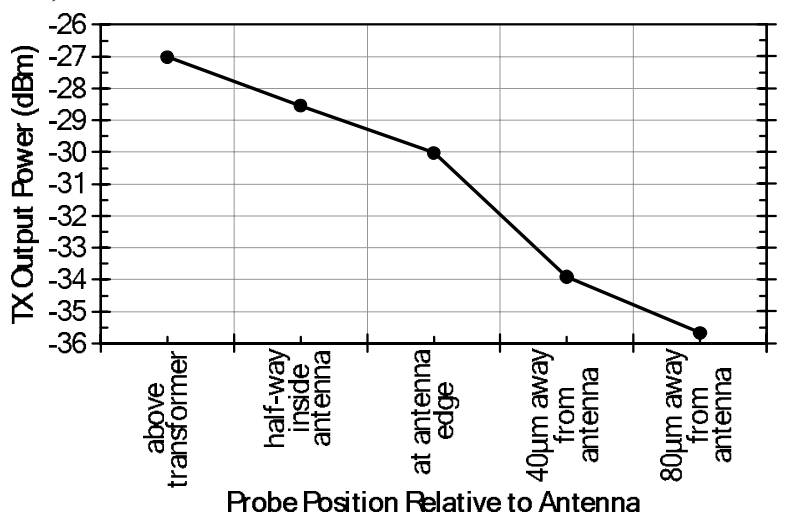

Fig. 13. Output power of the transmitter with antenna, measured at 5 different locations near the antenna.

\section{B. 170-GHz Transceiver}

The receiver antenna pattern of Fig. 11 was measured on the transceiver with on-die antennas using a probe suspended near the receiver antenna. The on-chip oscillator signal is at 165.3 GHz and the 162.6-GHz RF signal is applied through a GSG probe placed above the receive antenna. The receiver gain is compared in Fig. 12 with that measured on an identical receiver without on-die antennas. The DSB noise figure of the receiver with pads is $21 \mathrm{~dB}$. Fig. 13 shows the transmitter 
output power at $165.3 \mathrm{GHz}$, measured on the transceiver with antennas for several positions of the sensing probe above the transmitter antenna. For reference, the measured output power of the transmitter with pads is $-5 \mathrm{dBm}$. The total DC power consumption of the transceiver, including three $170-\mathrm{GHz}$ amplifiers, quadrature oscillator, mixer, and static frequency divider, is $800 \mathrm{~mW}$.

\section{170-GHz Antennas}

Antenna gain measurements are summarized in Fig. 14 versus frequency, and in Figs. 15-17 at $164 \mathrm{GHz}$. The plotted antenna radiation pattern is calculated by subtracting the setup losses from the power measured by the sensing probe above the antenna and includes propagation loss. These results demonstrate that, although all antennas have significant loss, the dipoles perform better than the patch. The dipole with metal fill shows comparable or better performance than the conventional dipole while occupying significantly less area and satisfying all metal density rules.

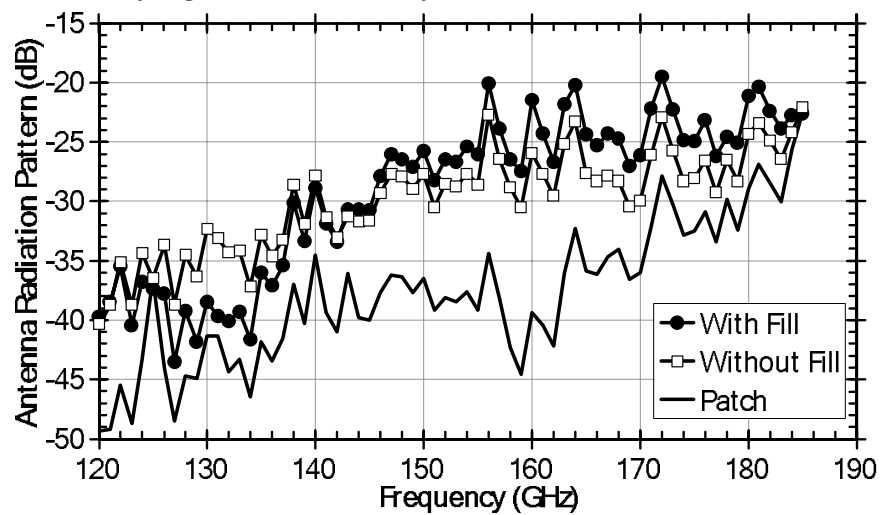

Fig. 14. Measured radiation pattern of the three antennas versus frequency.
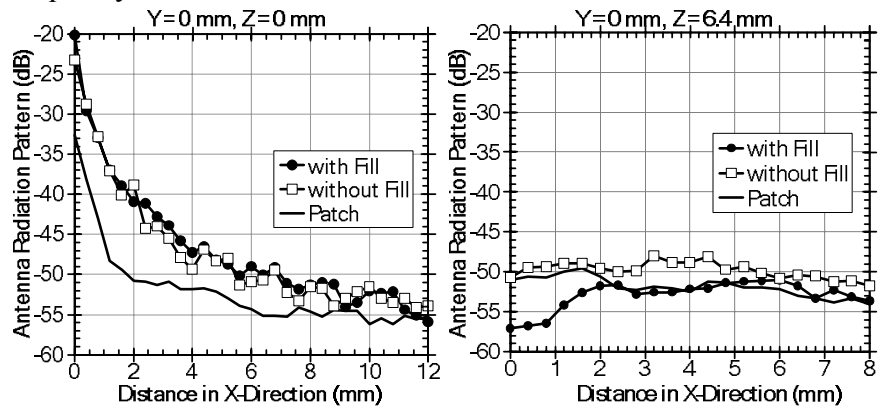

Fig. 15. Antenna radiation pattern at $164 \mathrm{GHz}$ measured by moving the probe away from the antenna in the X-direction for $\mathrm{Z}=0 \mathrm{~mm}$ (left) and $\mathrm{Z}=6.4 \mathrm{~mm}$ (right), $\mathrm{Y}=0 \mathrm{~mm}$ in both cases.

\section{CONCLUSION}

The first single-chip silicon transceiver with on-die transmit and receive antennas operating in the $160-\mathrm{GHz}$ to $180-\mathrm{GHz}$ range was demonstrated. Antenna test structure measurements indicate that a dipole design with floating metal strips has better performance than a patch antenna while satisfying the stringent metal density rules of 130-nm CMOS and SiGe BiCMOS technologies. Nevertheless, the antenna loss remains unacceptably high, around $25 \mathrm{~dB}$, indicating that a thickmetal, thick low-k dielectric BEOL and a low-k sealant may be needed to alleviate it.
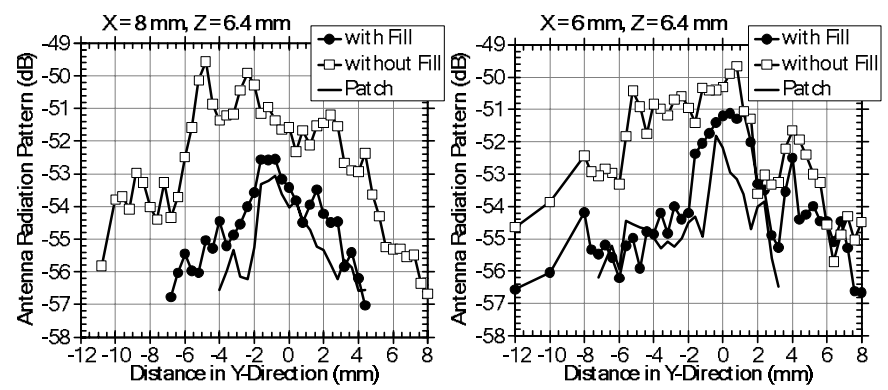

Fig. 16. Antenna radiation pattern at $164 \mathrm{GHz}$ measured by moving the probe around the antenna in the Y-direction for $X=8 \mathrm{~mm}$ (left) and $\mathrm{X}=6 \mathrm{~mm}$ (right), $\mathrm{Z}=6.4 \mathrm{~mm}$ in both cases.
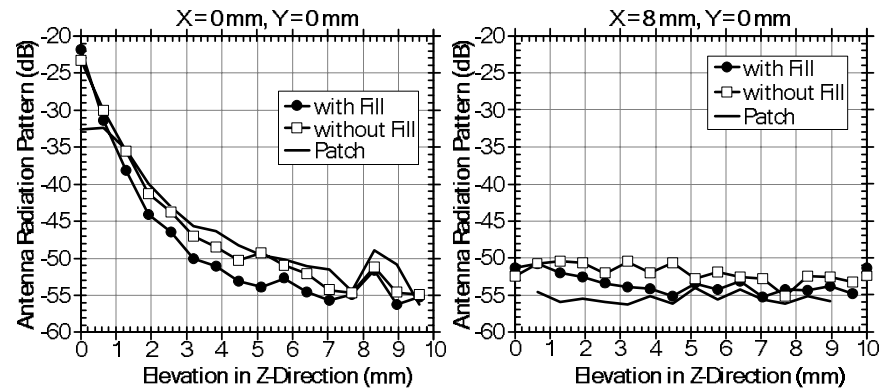

Fig. 17. Antenna radiation pattern at $164 \mathrm{GHz}$ measured by moving the probe around the antenna in the Z-direction for $X=0 \mathrm{~mm}$ (left) and $\mathrm{X}=8 \mathrm{~mm}$ (right), $\mathrm{Y}=0 \mathrm{~mm}$ in both cases.

\section{ACKNOWLEDGEMENT}

The authors thank STMicroelectronics for technology access, NSERC for funding, CMC and Jaro Pristupa for CAD support.

\section{REFERENCES}

[1] E. Laskin, et al, "80/160-GHz Transceiver and $140 \mathrm{GHz}$ Amplifier in SiGe Technology," IEEE RFIC, pp.153-156, 2007.

[2] A. Babakhani, et al, "A 77-GHz phased-array transceiver with on-chip antennas in silicon: Receiver and antennas," IEEE JSSC, vol. 41, pp. 2795-2806, Dec. 2006.

[3] E. Laskin, et al, "Low-Power, Low-Phase Noise SiGe HBT Static Frequency Divider Topologies up to $100 \mathrm{GHz}$," IEEE BCTM, pp. 235-238, Oct. 2006.

[4] T.O. Dickson, et al, "30-100-GHz Inductors and Transformers for Millimeter-Wave (Bi)CMOS Integrated Circuits," IEEE MTT, vol. 53, no. 1, pp. 123-133, Jan 2005.

[5] T.S.D. Cheung, et al, "On-chip interconnect for mm-wave applications using an all-copper technology and wavelength reduction," IEEE ISSCC, pp. 396-397, Feb. 2003.

[6] P. Chevalier, et al, "High-Speed SiGe BiCMOS Technologies: 120-nm Status and End-of-Roadmap Challenges," IEEE SiRF, pp. 18-23, Jan. 2007 